\title{
Identificación de mega-ambientes para potenciar el uso de genotipos superiores de arroz en Panamá
}

\author{
Ismael Camargo-Buitrago(1), Evelyn Quirós-Mc Intire(1) y Román Gordón-Mendoza(1)
}

\begin{abstract}
(1)Instituto de Investigación Agropecuaria de Panamá, Apartado Postal 0819-05850, El Dorado, Panamá 6A, Panamá. E-mail: icamargo@cwpanama.net, evelynitzel26@gmail.com, gordon.roman@gmail.com
\end{abstract}

\begin{abstract}
Resumen - El objetivo de este trabajo fue evaluar tres métodos para identificar mega-ambientes, para optimizar el uso del potencial genético de los cultivares de arroz, durante el proceso de selección, y para hacer recomendaciones sobre siembras comerciales en Panamá. Los datos experimentales fueron obtenidos de los ensayos de productividad de cultivares precoces realizados entre 2006 y 2008. Para lograr la estratificación de los ambientes y definir los mega-ambientes, se utilizaron los métodos del genotipo vencedor mediante el modelo AMMI1, el modelo biplot GGE y el de conglomerado por el método de Ward, complementado con el biplot GGE. Los tres métodos utilizados identificaron dos mega-ambientes, donde los cultivares sobresalientes fueron Fedearroz 473 e Idiap 145-05. Hubo una coincidencia de 100\% en el agrupamiento del conglomerado x el biplot GGE, mientras que entre conglomerado x AMMI1 y biplot GGE x AMMI1 fue de 95,2\%. El genotipo más estable, en ambos mega-ambientes, fue el cultivar Idiap 145-05, lo que indica capacidad de adaptación amplia y específica. La capacidad adaptativa de los genotipos superiores y no las condiciones agroclimáticas de las localidades evaluadas fue responsable de la definición de los mega-ambientes.
\end{abstract}

Términos para indexación: Oryza sativa, AMMI, biplot GGE, conglomerados, interacción genotipo x ambiente.

\section{Identifying mega-environments to enhance the use of superior rice genotypes in Panama}

\begin{abstract}
The objective of this work was to evaluate three methods to identify mega-environments, in order to optimize the use of the genetic potential of rice cultivars during the selection process and to make recommendations for commercial plantations in Panama. Experimental data were obtained from the test performance, between 2006 and 2008, for early maturing cultivars. To achieve the stratification of environments and define mega-environments, the winner genotype method by the AMMI1 model, GGE biplot model and cluster by Ward's method supplemented by GGE biplot were used. The three methods used identified two mega-environments, where the outstanding cultivars were Fedearroz 473 e Idiap 145-05. There was 100\% coincidence in the grouping of the cluster $\mathrm{x}$ the GGE biplot, with $95.2 \%$ coincidence between the AMMI1 $\mathrm{x}$ cluster and GGE biplot x AMMI1. The most stable genotype, in both mega-environments, was the Idiap 145-05 cultivar, which indicates its broad and specific adaptive capacity. The adaptive capacity of the superior genotypes and not the agroclimatic conditions of the assessed localities was responsible for defining the mega-environments.
\end{abstract}

Index terms: Oryza sativa, AMMI, GGE biplot, cluster, genotype x environment interaction.

\section{Introducción}

Para maximizar la expresión del potencial de productividad de los cultivares, cuando existen regiones heterogéneas para la producción, a menudo es conveniente subdividir una región con ambientes heterogéneos en varios mega-ambientes relativamente homogéneos, con el objetivo de seleccionar cultivares adaptadas para cada mega-ambiente. En las regiones tropicales como Panamá, en años diferentes, las mismas localidades presentan condiciones agroecológicas distintas, especialmente en cuanto a la disponibilidad de humedad en el suelo, producto de la precipitación pluvial, que en el caso de los cultivos plantados bajo condiciones de secano constituyen un factor limitante en la producción de grano (Gordón et al., 2005a, 2005b). Por ello, los ensayos para evaluar los genotipos experimentales y comerciales deben realizarse todos los años para explorar esta variabilidad ambiental, lo que se refleja en la respuesta diferencial de los genotipos en los ambientes de prueba. 
Los efectos principales del genotipo (G), el ambiente (E) y la interacción genotipo $\mathrm{x}$ ambiente han sido estudiados para estimar y definir la estabilidad de cultivares (Yan \& Tinker, 2005; Gauch Junior, 2006; Glaz \& Kang, 2008). Además, diversos autores desarrollaron métodos para el agrupamiento de los ambientes (Gauch Junior \& Zobel, 1997; Annicchiarico et al., 2000; Atlin et al., 2000; Trethowan et al., 2003; Yang et al., 2005).

El modelo AMMI, propuesto por Zobel et al. (1988) y validado por Gauch Junior \& Zobel (1989) y Crossa et al. (1990), combina el análisis de regresión lineal con el análisis por componentes principales (PCA), bajo los supuestos que los efectos principales (genotipo y ambiente) son de naturaleza aditiva y la interacción genotipo $\mathrm{x}$ ambiente es de naturaleza multiplicativa.

El modelo biplot GGE es una herramienta que ha incrementado su popularidad entre los mejoradores de plantas y otros investigadores agrícolas y es muy utilizada en la evaluación de cultivares y en la investigación de mega-ambientes, pues permite examinar, mediante la interacción genotipo $\mathrm{x}$ ambiente, la capacidad de discriminar y la representatividad de los ambientes de prueba como una medida conveniente para definir mega-ambientes homogéneos (Yan et al., 2000; Yan \& Hunt, 2002; Yan \& Kang, 2003).

El análisis de conglomerado mediante el método de Ward es una técnica multivariada, que busca agrupar elementos (o variables) y que trata de lograr la máxima homogeneidad en cada grupo y la mayor diferencia entre los grupos (Johnson, 2000).

Sin embargo, relativamente pocos investigadores han estudiado la interacción genotipo $\mathrm{x}$ ambiente, para determinar las ventajas o conveniencia de utilizar algunos ambientes de prueba, para optimizar recursos y efectuar recomendaciones más precisas. Diversos autores (Gauch Junior \& Zobel, 1997; Ebdon \& Gauch, 2002; Samonte et al., 2005; Yan \& Tinker, 2005; Felipe et al., 2010) propusieron identificar mega-ambientes por el modelo AMMI, aplicando el concepto de "genotipo ganador" (vencedor o superior), o sea, aquel que alcanza la producción más alta en uno o varios ambientes. Gauch Junior \& Zobel (1997) plantean que las estrategias estadísticas para la identificación de los mega-ambientes debe cumplir algunos criterios como: la flexibilidad en el manejo de los ensayos de productividad con varios diseños, centrándose en la fracción de la variación total, que es relevante para la identificación de los mega-ambientes; la dualidad en brindar información integrada, tanto de genotipos como ambientes; y la relevancia para cumplir el objetivo principal de destacar los genotipos ganadores. En la investigación agrícola, diferentes conceptos y definiciones de estabilidad han sido desarrollados para aplicaciones en programas de mejoramiento genético, donde se conducen ensayos de productividad (Lin et al., 1986; Becker \& León, 1988).

El objetivo de este trabajo fue comparar tres métodos para identificar mega-ambientes y para optimizar el uso del potencial genético de los cultivares de arroz en el proceso de selección y en siembras comerciales en Panamá.

\section{Materiales y Métodos}

Los datos experimentales para este estudio fueron obtenidos de la base de datos de los ensayos de evaluación de cultivares de ciclo precoz, realizados en las principales zonas productoras de arroz en el sistema de secano, por el proyecto de investigación e innovación para el desarrollo de germoplasma mejorado de arroz, del Instituto de Investigación Agropecuaria de Panamá (IDIAP), entre los años 2006 y 2008. Los ambientes de prueba, ubicación geográfica y fecha de plantío se presentan en el Cuadro 1. Los cultivares evaluadas fueron Idiap 145-05 (IDIAP-FLAR), Idiap 54-05 (IDIAP-FLAR), Fedearroz 473 (FEDEARROZ), Colombia XXI (FEDEARROZ), Oryzica 1 (ICA-CIAT), RHP-6-06 (IDIAP-FLAR), RHP-7-06

Cuadro 1. Ambientes, ubicación geográfica y fecha de plantío, durante el periodo de evaluación, para la identificación de mega-ambientes para cultivo de arroz.

\begin{tabular}{llccc}
\hline Ambiente & Coordenadas geográficas & \multicolumn{3}{c}{ Fecha de plantío } \\
\cline { 3 - 5 } & & 2006 & 2007 & 2008 \\
\hline Alanje & $08^{\circ} 23^{\prime} 43^{\prime \prime} \mathrm{N}, 82^{\circ} 33^{\prime} 34 " \mathrm{~W}$ & $21 / 8$ & $9 / 9$ & $21 / 7$ \\
Barú & $08^{\circ} 27^{\prime} 04^{\prime \prime} \mathrm{N}, 82^{\circ} 51^{\prime} 22^{\prime \prime} \mathrm{W}$ & $25 / 8$ & $10 / 9$ & $28 / 7$ \\
Remedios & $08^{\circ} 11^{\prime} 44^{\prime \prime} \mathrm{N}, 81^{\circ} 49^{\prime} 57^{\prime \prime} \mathrm{W}$ & - & $21 / 8$ & - \\
Soná & $07^{\circ} 47^{\prime} 56^{\prime \prime} \mathrm{N}, 81^{\circ} 15^{\prime} 78^{\prime \prime} \mathrm{W}$ & $1 / 8$ & $1 / 8$ & $28 / 7$ \\
Divisa & $07^{\circ} 47^{\prime} 65^{\prime \prime} \mathrm{N}, 81^{\circ} 15^{\prime} 75^{\prime \prime} \mathrm{W}$ & - & $5 / 9$ & $14 / 7$ \\
Calabacito & $08^{\circ} 07^{\prime} 33^{\prime \prime} \mathrm{N}, 80^{\circ} 41^{\prime} 32^{\prime \prime} \mathrm{W}$ & $1 / 8$ & - & - \\
Penonomé & $08^{\circ} 05^{\prime} \mathrm{N}, 80^{\circ} 02^{\prime} \mathrm{W}$ & - & $28 / 7$ & $16 / 7$ \\
Tonosí & $07^{\circ} 23^{\prime} 23^{\prime \prime} \mathrm{N}, 80^{\circ} 27^{\prime} 43^{\prime \prime} \mathrm{W}$ & - & $7 / 9$ & $3 / 9$ \\
Tanara & $09^{\circ} 07^{\prime} 44 " \mathrm{~N}, 79^{\circ} 13^{\prime} 46^{\prime \prime} \mathrm{W}$ & $1 / 8$ & $18 / 7$ & - \\
Changuinola & $07^{\circ} 47^{\prime} 56^{\prime \prime} \mathrm{N}, 81^{\circ} 15^{\prime} 78^{\prime \prime} \mathrm{W}$ & $7 / 9$ & - & $24 / 10$ \\
\hline
\end{tabular}

Fuente: base de datos del proyecto de investigación e innovación: Desarrollo de germoplasma mejorado de arroz (Instituto de Investigación Agropecuaria de Panamá, 2010). 
(IDIAP-FLAR), FLAR 179-00 (IDIAP-FLAR), FLAR 195-00 (IDIAP-FLAR) y FLAR 198-00 (IDIAP-FLAR). Se observa que la fecha de plantío en una misma localidad es muy variable de un año para otro. El diseño experimental fue de bloques completos al azar con tres repeticiones, y la unidad experimental constó de 10 hileras de cinco metros de longitud, separadas en $20 \mathrm{~cm}$, para un área total de $10 \mathrm{~m}^{2}$; la parcela efectiva fue de $4,8 \mathrm{~m}^{2}$, con seis hileras de cuatro metros de longitud. Se hicieron controles de malezas, conforme al complejo prevaleciente, y la fertilización se efectuó de acuerdo al análisis de suelo. No se hicieron controles de plagas y enfermedades. Los datos obtenidos de la productividad de grano limpio y seco al $14 \%$ de humedad se expresan en megagramos por hectárea $\left(\mathrm{Mg} \mathrm{h}^{-1}\right)$. El análisis estadístico de los datos consistió en la aplicación de técnicas multivariadas como: AMMI, biplot GGE y método de agrupamiento de Ward (Johnson, 2000).

El análisis AMMI para productividad de grano de arroz fue realizado con el programa SAS (SAS Institute, 2002), de acuerdo con Hernández \& Crossa (2000).

La estratificación de los ambientes fue estimada por medio del concepto de genotipos vencedores, de acuerdo a la propuesta de Gauch Junior \& Zobel (1997) validada por Ebdon \& Gauch Junior (2002). Se define un genotipo vencedor como aquel que alcanza la productividad más alta en un determinado ambiente. Las estimativas de AMMI1 (modelo que considera solamente el primer eje del componente principal) y de la productividad de cada genotipo, en cada ambiente $(\widetilde{Y} i j)$, se obtuvieron mediante la siguiente ecuación:

$$
\tilde{\mathrm{Y}}_{\mathrm{ij}}=\overline{\mathrm{Y}}_{\mathrm{i}}+\left(\operatorname{IPCA} 1_{\mathrm{i}}\right)\left(\operatorname{IPCA} 1_{\mathrm{j}}\right)
$$

en que: $\bar{Y}_{i}=$ es la media del genotipo i sobre todos los ambientes; IPCA $1_{i}$ es la puntuación del genotipo $i$ en el primer eje principal de la interacción (IPCA1), estimado en el análisis de componentes principales; y IPCA $1_{\mathrm{j}}$ es la puntuación del ambiente $\mathrm{j}$, en el mismo eje (IPCA1).

De esta manera, la productividad de cada genotipo, en cada ambiente $\left(\widetilde{\mathrm{Y}}_{\mathrm{ij}}\right)$, es representada como una función lineal simple de la puntuación ambiental $\left(\mathrm{IPCA} 1_{\mathrm{j}}\right)$, o sea una ecuación recta con intercepto $\left(\mathrm{Y}_{\mathrm{i}}\right)$ e inclinación igual a la puntuación genotípica de la interacción (IPCA $1_{i}$ ). El punto de cruzamiento entre las dos rectas correspondientes a los genotipos ganadores define la transición adaptativa de los dos genotipos y, simultáneamente, dos estratos ambientales (mega-ambientes) de adaptación productiva.

Para definir los mega-ambientes, los datos fueron analizados por el método biplot GGE, utilizándose el software "Visual statistical analysis system GGE biplot pattern explorer" versión 6.3 (Yan, 2009). Mediante los procedimientos gráficos mean $\mathrm{x}$ stability y la gráfica "which won where/what" (genotipos vencedores), se aprecia de manera visual y efectiva la evaluación de cultivares y la determinación de mega-ambientes (Yan et al., 2000).

Los datos de productividad fueron sometidos a un análisis de conglomerado mediante el método de Ward (Johnson, 2000). Para discernir el comportamiento de los genotipos, los ambientes y la interacción, dentro de cada mega-ambiente, el análisis de conglomerado fue complementado con el biplot GGE, para obtener el comportamiento relativo de cada genotipo en los dos mega-ambientes, para facilitar la interpretación de la interacción genotipo $\mathrm{x}$ ambiente. En este método, se busca la mínima variabilidad dentro de los conglomerados; es entonces un problema de optimización. Este método se basa en la pérdida de información resultante al agrupar los ítems en grupos (Johnson, 2000), la cual está medida por la suma total al cuadrado de las desviaciones de cada objeto del centroide del grupo al que pertenece. La suma de cuadrados se calcula mediante la expresión:

$$
\mathrm{SCW}=\frac{1}{\left(1 / \mathrm{n}_{\mathrm{h}}+1 / \mathrm{n}_{\mathrm{i}}\right)}\left\|\mathrm{X}_{\mathrm{h}}-\mathrm{X}_{\mathrm{i}}\right\|^{2}
$$

en que: $\mathrm{X}_{\mathrm{h}} \mathrm{y} \mathrm{X}_{\mathrm{i}}$ son los centroides y $\mathrm{n}_{\mathrm{h}} \mathrm{y} \mathrm{n}_{\mathrm{i}}$ son los tamaños de los conglomerados h y i, respectivamente.

Para un único atributo o variable, la suma de cuadrados se obtiene como:

$$
\mathrm{SCW}=\sum_{\mathrm{j}=1}^{\mathrm{k}}\left[\sum_{\mathrm{i}=1}^{\mathrm{n}_{j}} \mathrm{X}_{\mathrm{ij}}^{2}-\frac{1}{\mathrm{n}_{\mathrm{j}}}\left(\sum_{\mathrm{i}=1}^{\mathrm{n}_{\mathrm{j}}} \mathrm{X}_{\mathrm{ij}}\right)^{2}\right]
$$

en que: $X_{\mathrm{ij}}$ es el valor del atributo para el i-ésimo individuo, en el j-ésimo conglomerado; $\mathrm{k}$ es el número del conglomerado en cada etapa; $y n_{j}$ es el número de individuos para el j-ésimo conglomerado. En el primer paso, se empieza con $\mathrm{n}$ grupos, un caso por grupo, $\mathrm{y}$ aquí la suma de cuadrados de Ward (SCW) es cero. En el segundo paso, se buscan los dos casos que produzcan el menor incremento en la suma de cuadrados, dentro de todas las posibles combinaciones de dos objetos. En el tercer paso, se toman los ( $\mathrm{n}-1)$ grupos conformados, 
se calcula la SCW y se juntan aquellos que produzcan el menor incremento en la variabilidad. El proceso continua hasta obtener un grupo de $\mathrm{n}$ objetos casos.

\section{Resultados y Discusión}

La productividad de grano fue significativamente afectada por los efectos ambientales y genotípicos, los cuales explican, respectivamente, el 53,2 y 7,63\% de la suma de cuadrado total (Cuadro 2). La interacción genotipo x ambiente expresa el 16,4\% de la variación para productividad de grano. La partición de la interacción a través del modelo AMMI, muestra que los dos primeros ejes del componente principal (PCA 1 y PCA 2) explican, respectivamente, el 33,5 y 17,1\% de la suma de cuadrados de la interacción, y totalizan $50,6 \%$ de la suma de cuadrados de la interacción genotipo x ambiente. El tercer eje (PCA 3) explica el $16,7 \%$.

La diferencia entre los cultivares, en relación a la dirección y magnitud entre el eje de la productividad y el PCA 1, es importante (Gauch Junior \& Zobel, 1989). El cultivar superior es aquel con alta productividad de grano y estabilidad a través de los ambientes de prueba. Los cultivares con mejor productividad fueron Fedearroz 473, con aparente adaptación a los ambientes favorables, y Idiap 145-05; ambas se diferencian en su estabilidad. El cultivar Idiap 145-05 fue el más estable a través de los ambientes y seleccionado como el más apropiado entre los cultivares evaluados (Figura 1). El modelo AMMI puede, además, ser utilizado para identificar apropiadamente cultivares testigos para

Cuadro 2. Análisis de varianza AMMI y significancia de los efectos de genotipo, ambiente, genotipo $\mathrm{x}$ ambiente, respecto a productividad de grano de arroz y a la participación de interacción genotipo x ambiente, en los primeros ejes del componente principal ${ }^{(1)}$.

\begin{tabular}{lccccccc}
\hline $\begin{array}{l}\text { Fuente de } \\
\text { variación }\end{array}$ & GL & SC & CM & F & P & $\begin{array}{c}\text { SC } \\
\text { G x A (\%) }\end{array}$ & $\begin{array}{c}\text { Acumulado SC } \\
\text { G x A (\%) }\end{array}$ \\
\hline Ambiente (A) & 20 & 415,929 & 20,7965 & 16,810 & $* *$ & - & - \\
Genotipo (G) & 9 & 59,590 & 6,6211 & 19,930 & $* *$ & - & - \\
Gx A & 180 & 128,197 & 0,7122 & 2,140 & $* *$ & - & - \\
PCA1 & 28 & 43,002 & 1,5358 & 3,714 & $* *$ & 33,5 & 33,5 \\
PCA2 & 26 & 21,987 & 0,8456 & 2,045 & $*$ & 17,1 & 50,6 \\
PCA3 & 24 & 21,43180 & 0,892990 & 2,159 & $* *$ & 16,7 & 67,3 \\
\hline Error & 377 & 125,22171 & 0,332153 & & & & \\
Total & 629 & 781,49081 & & & & & \\
\hline
\end{tabular}

${ }^{(1)} \mathrm{GL}$, grados de libertad; SC, suma de cuadrados; CM, cuadrado medio. $*$ y **Significativo al 5 y $1 \%$ de probabilidad, por el test $\mathrm{F}$. todas las localidades o para localidades específicas (testigos específicos). En este sentido, los resultados sugieren que Idiap 145-05 puede ser el cultivar testigo para todos los ambientes, por su estabilidad en la productividad.

En este estudio, la estabilidad de los cultivares, basada en el valor absoluto del PCA 1 (AMMI1), tiene el siguiente orden: Idiap 145-05 (0,062), FLAR 179-00 (0,074), RHP-6-06 (0,147), FLAR 198-00 (-0,159), FLAR 195-00 (0,185), Oryzica $1(0,198)$, RHP-7-06 $(-0,263)$, Colombia XXI $(-0,371)$, Idiap 54-05 (-1,025) y Fedearroz $473(1,549)$.

La definición de mega-ambientes mediante la estratificación ambiental, de acuerdo al modelo AMMI1, indica que los cultivares ganadores fueron Fedearroz 473 e Idiap 145-05, en 18 de los 21 ambientes de prueba entre 2006 y 2008 (Figura 2). Estos cultivares presentan adaptación específica al estrato o mega-ambiente donde fueron vencedoras $y$, simultáneamente, estabilidad en sentido agronómico para las localidades que componen el mega-ambiente (Felipe et al., 2010). El cultivar Fedearroz 473 tuvo el mejor comportamiento en 11 ambientes (Calabacito 2007, Penonomé 2007, Tonosí 2007, Barú 2007, Barú 2006, Soná 2007, Remedios 2007, Soná 2006, Divisa 2007, Tanara 2006 y Changuinola 2006), mientras que Idiap 145-05 lo fue en diez sitios (Changuinola 2008, Penonomé 2008, Soná 2008, Tonosí 2008, Barú 2008, Divisa 2008, Alanje 2007, Alanje 2006, Tanara 2007 y Alanje 2008).

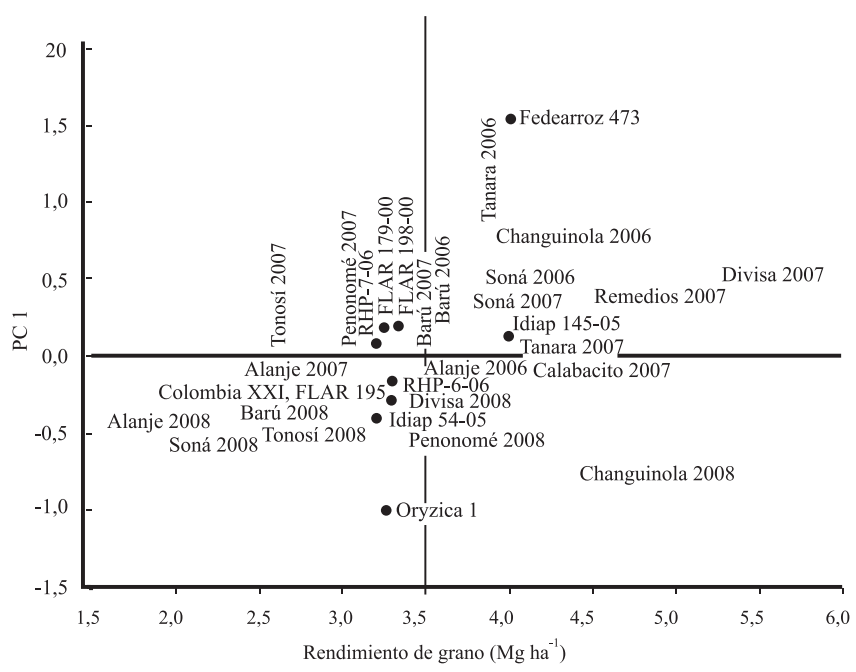

Figura 1. AMMI biplot con los efectos principales de genotipo de arroz y del ambiente (media de productividad de grano x las puntuaciones del PCA 1; AMMI1). 
Cada cultivar ganador determina una estratificación ambiental o mega-ambiente e indica la propia potencial adaptabilidad específica (Figura 2). La línea punteada vertical identifica la transición entre los dos estratos ambientales y corresponde al punto donde se cruzan los cultivares ganadores (Gauch Junior \& Zobel, 1997; Samonte et al., 2005; Felipe et al., 2010). La formación de estos mega-ambientes puede deberse a la adaptabilidad amplia de los cultivares vencedores y no a la agrupación de los ambientes por similaridad agroclimática (suelos, precipitación etc.), debido a que las condiciones ambientales en una misma zona geográfica pueden variar significativamente de un ciclo agrícola a otro.

La conducción de ensayos en múltiples localidades, durante varios años, es necesaria para caracterizar los patrones de respuesta de las localidades agrupadas en regiones y para identificar cultivares con alta productividad de grano para cada región (Yan et al., 2000; Yan \& Rajcan, 2002).

En la gráfica biplot GGE (Figura 3), los cultivares ubicados en el vértice derecho del cuadrante representan aquellos con mayor productividad de grano y están altamente relacionadas con los ambientes cercanos: son los cultivares superiores Fedearroz 473 e Idiap 145-05. Las localidades se agrupan en dos mega-ambientes, el primero dominado por Idiap 145-05 y el segundo por Fedearroz 473. Los otros cultivares del vértice,

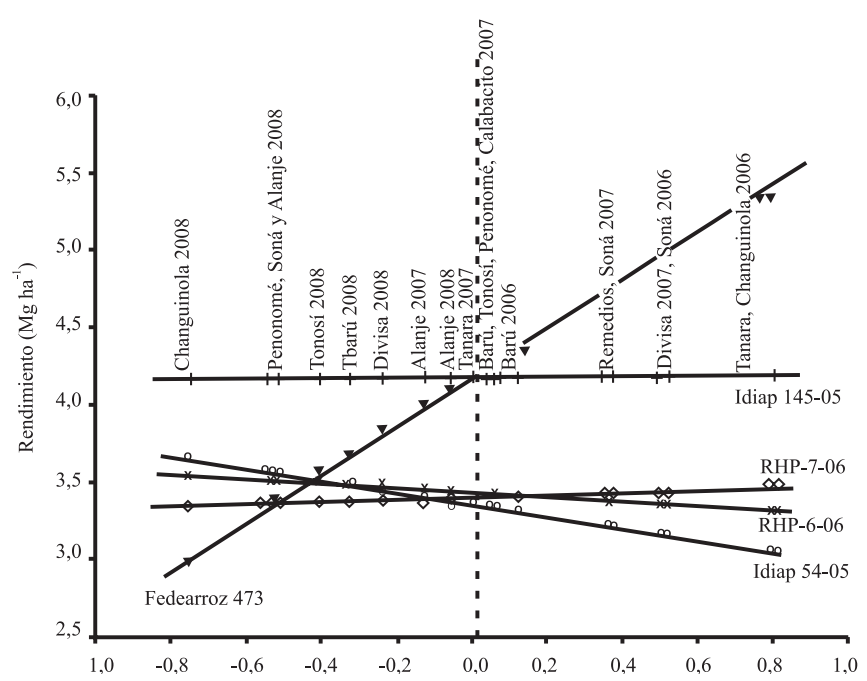

Figura 2. Respuestas genotípicas de las cultivares de arroz encuanto a la productividad de grano y estratificación de mega-ambientes, en función de las puntuaciones ambientales estimadas por el modelo AMMI (IPCA1). ubicadas en el lado izquierdo y alejadas de los vectores de las localidades presentaron un pobre desempeño, y están bien alejadas de la posición de las localidades.

Los cultivares estables tienen altas puntuaciones en el PCA 1, lo que significa altas productividades, y bajas puntuaciones en el PCA 2 son indicativas de alta estabilidad; de modo similar, los ambientes con bajas puntuaciones en el PCA 2 tienen más representatividad, $\mathrm{y}$ altas puntuaciones en el PCA 1 indican mayor capacidad discriminatoria de genotipos en términos del efecto principal genotípico (Yan et al., 2000; Yan \& Rajcan, 2002).

El análisis mean x stability del biplot GGE (Figura 4) proyecta la clasificación relativa de los cultivares basado en el eje del comportamiento promedio de los ambientes, este eje determina la respuesta en adaptabilidad y estabilidad; en cuanto a la adaptabilidad, los genotipos siguen el siguiente orden decreciente: Fedearroz 473, Idiap 145-05, RHP-7-06, Oryzica 1, FLAR 179-00, FLAR 198-00, RHP-6-06, Colombia XXI, RHP-8-06, FLAR 195-05 e Idiap 54-05. Sin embargo, en relación a la estabilidad, los genotipos que están más próximos al eje medio ambiental, son los más estables y, de esta manera, siguen en orden decreciente, en que las más estables son: FLAR 195-05, Idiap 54-05, FLAR 179-05, RHP-6-06, Colombia XXI, RH7, FLAR 198-00, Idiap 145-05, Fedearroz 473 y Oryzica 1. En este estudio, Idiap 145-05 y Fedearroz 473 mantuvieron altas productividades, pero esta última presentó baja

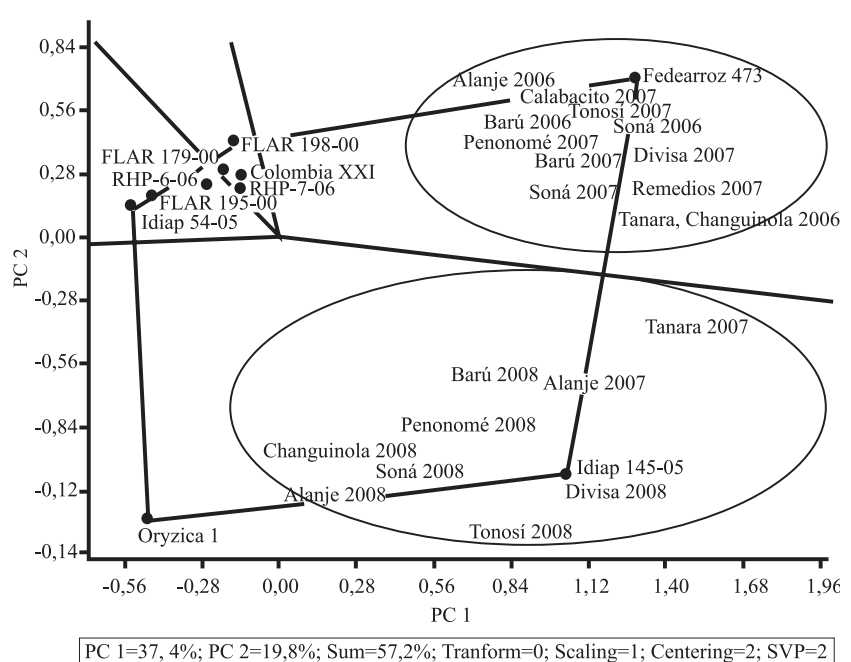

Figura 3. Polígono del biplot GGE que identifica dos mega-ambientes y sus respectivos genotipos superiores de arroz (Fedearroz 473 e Idiap 145-05). 
estabilidad. Así, el cultivar Idiap 145-05 se muestra como la de mejor comportamiento respecto a su productividad y su estabilidad de manera conjunta. Para la identificación de los cultivares superiores (estabilidad y adaptabilidad), basada en el modelo AMMI (Zobel et al., 1988) y en el biplot GGE (Yan et al., 2000), existe una correlación ( $\left.r>0,95^{* *}\right)$ de la puntuación del PCA 1 entre genotipo y la productividad promedio de grano a través de las localidades (Yan et al., 2000, 2001; Crossa et al., 2002; Yan \& Rajcan, 2002). En el presente estudio, la correlación PCA1 x productividad fue alta $(\mathrm{r}=0,974 * *)$.

Los métodos de análisis por conglomerado, visualizados mediante los dendrogramas, pueden ser de utilidad para agrupar los ambientes. El dendrograma concentra las localidades en dos mega-ambientes (Figura 5); en el primero, conformado por los sitios Barú 2006, Penonomé 2007, Alanje 2006, Calabacito 2007, Soná 2006, Remedios 2007, Soná 2007, Divisa 2007, Tanara 2006, Changuinola 2006, Barú 2007 y Tonosí 2007, mientras el segundo está integrado por Alanje 2007, Divisa 2008, Tonosí 2008, Soná 2008, Penonomé 2008, Tanara 2007, Barú 2008, Alanje 2008 y Changuinola 2008; en este grupo, entre las distancias más cercanas estuvieron las localidades

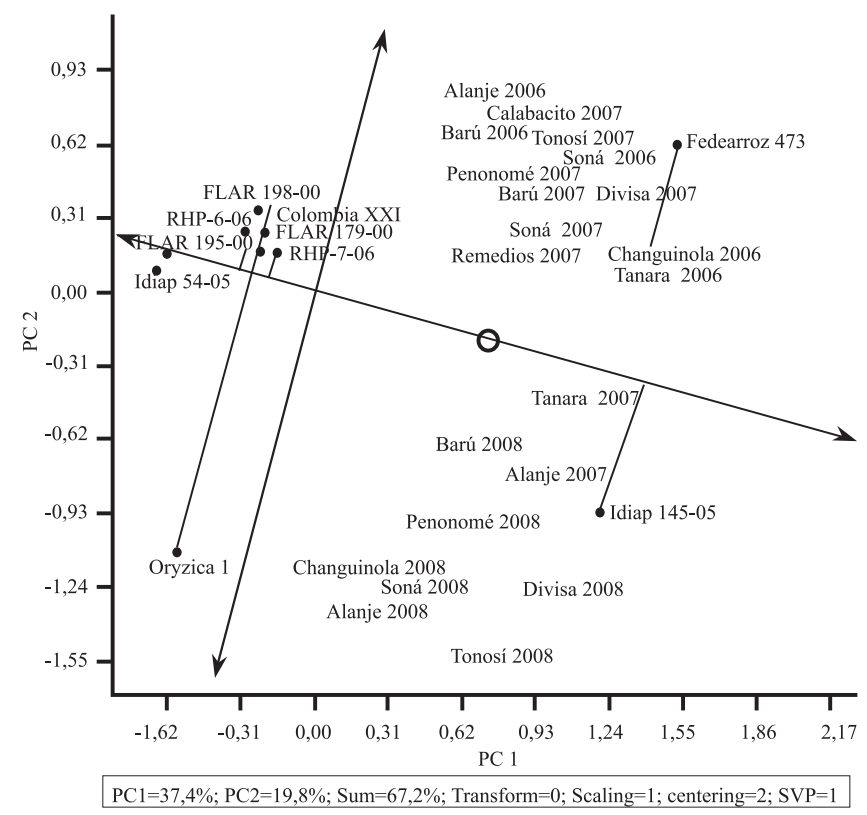

Figura 4. Media ambiental del biplot GGE, con definición de los mega-ambientes y sus respectivos genotipos superiores de arroz (Fedearroz 473 e Idiap 145-05). PC 1, comportamiento medio de los ambientes; PC 2 estabilidad.
Divisa 2008 y Tonosí 2008, seguidas de Alanje 2008 y Changuinola 2008. Al aplicar el análisis Biplot GGE al mega-ambiente 1 del dendrograma, obtenemos la Figura 6, que muestra primero las localidades que componen este mega-ambiente: Barú 2006, Penonomé 2007, Alanje 2006, Calabacito 2007, Soná 2006, Remedios 2007, Soná 2007, Divisa 2007, Tanara 2006, Changuinola 2006, Barú 2007 y Tonosí 2007. En seguida, se muestra la posición relativa de los cultivares evaluados, en función del eje del comportamiento medio de los ambientes, en que sobresale Fedearroz 473, y continuando en orden decreciente, Idiap 145-05, FLAR 198-00, Colombia XXI, RHP-6-06, FLAR 179-00, RHP-7-06, Idiap 54-05, FLAR 195-00, y Oryzica 1. Los cultivares más estables fueron FLAR 195-05, Idiap 54-05 y

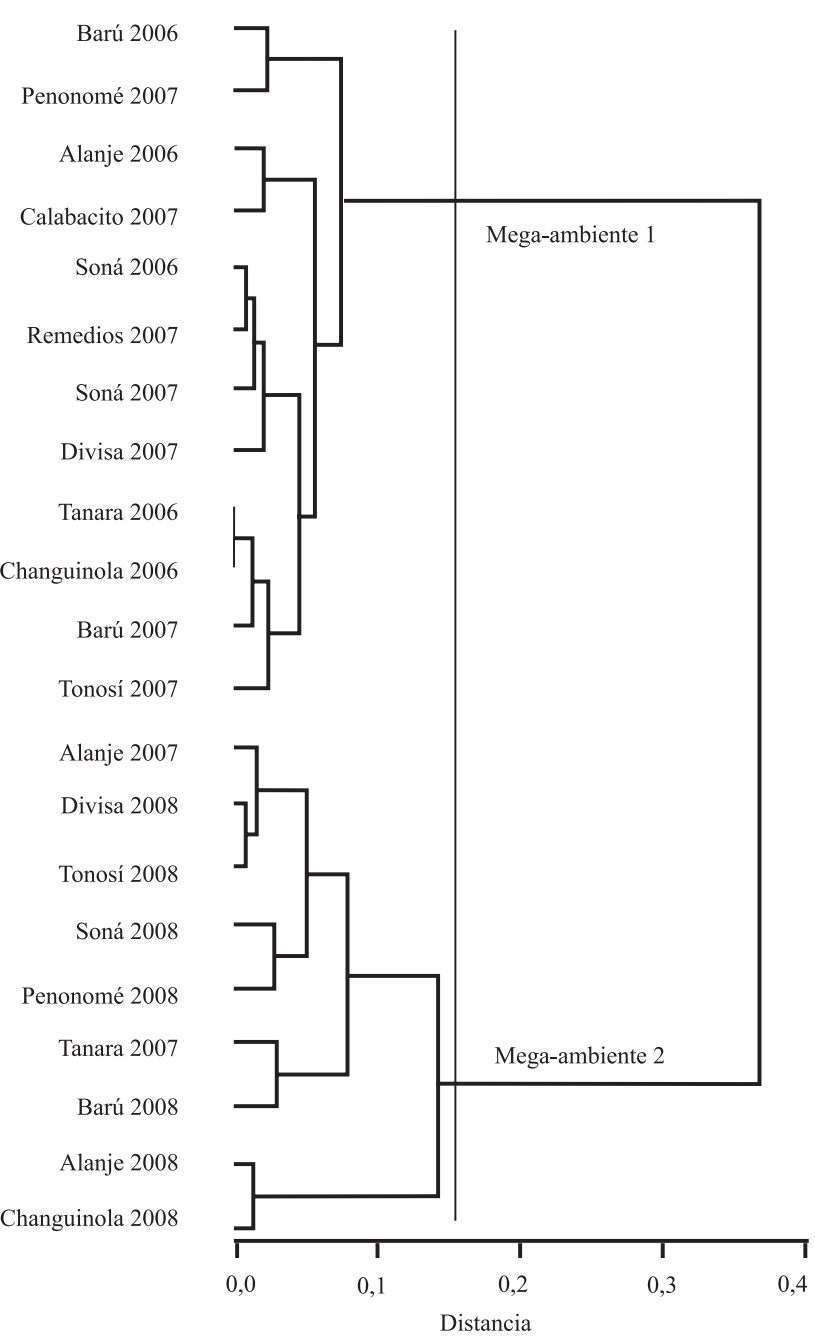

Figura 5. Conglomerado de los 21 ambientes evaluados por el método de WARD que agrupó las localidades en dos mega-ambientes. 
Oryzica 1, no obstante presentar bajas productividades y carecer de interés. Al considerar la productividad y la estabilidad, Fedearroz 473 e Idiap 145-05, con altas productividades de grano, presentaron también buena estabilidad; ambos cultivares representan excelentes opciones para siembras comerciales $\mathrm{y}$, como testigos, para la evaluación de nuevos cultivares en el futuro, en este mega-ambiente.

Sin embargo, la Figura 6 B, construida a partir del segundo agrupamiento del dendrograma (Figura 5),
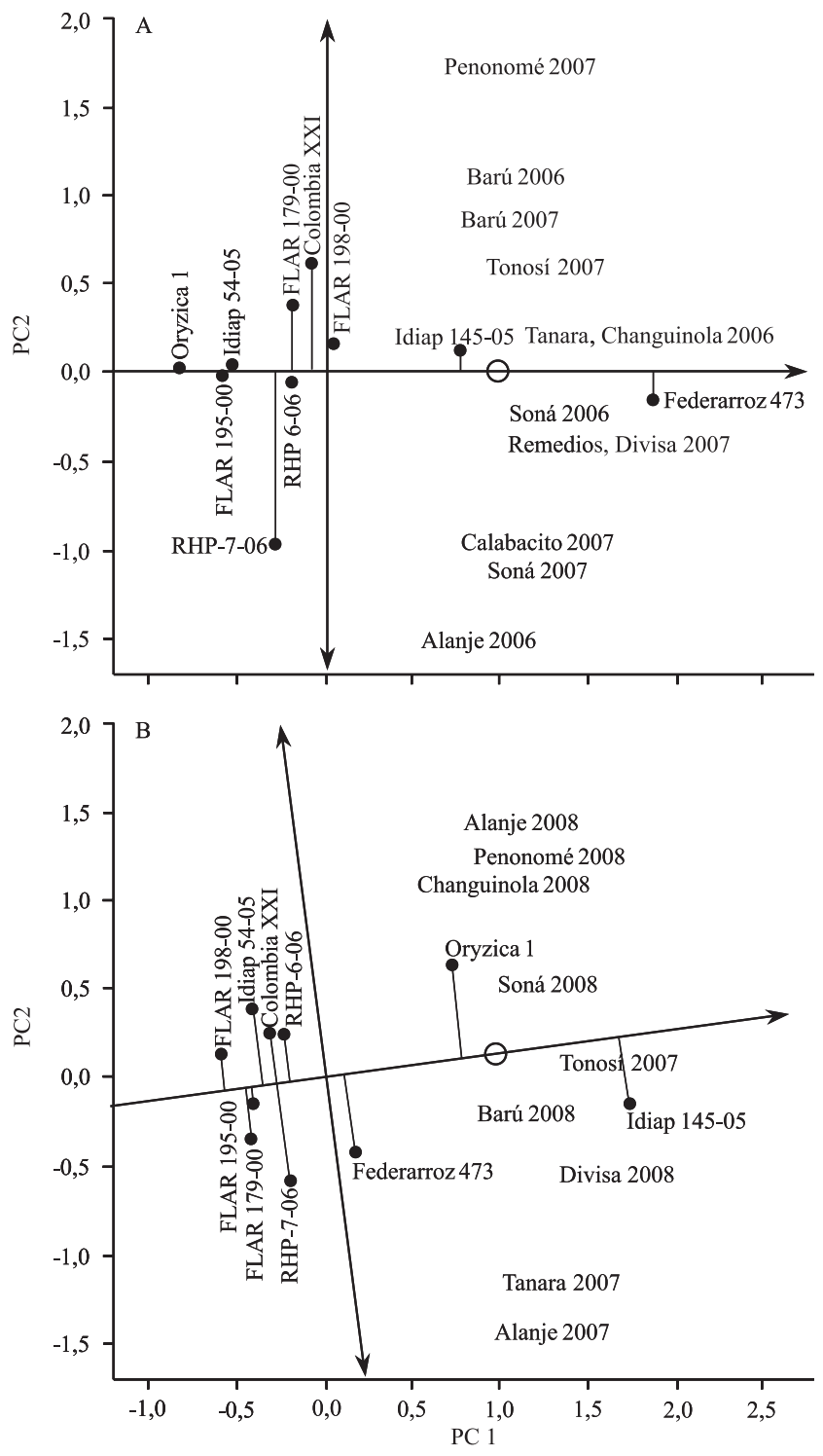

Figura 6. Media ambiental del biplot GGE, correspondiente a los agrupamientos del conglomerado, con los mega-ambientes, donde los genotipos de arroz superiores son: A, Fedearroz 473; B, Idiap 145-05. muestra las localidades que conforman este mega-ambiente: Alanje 2007, Divisa 2008, Tonosí 2008, Soná 2008, Penonomé 2008, Tanara 2007, Barú 2008, Alanje 2008 y Changuinola 2008. En la clasificación relativa de los cultivares, con base en el eje del comportamiento medio de los ambientes, el cultivar con mejor comportamiento en este mega-ambiente fue Idiap 145-05 y, en orden decreciente, Oryzica 1, Fedearroz 473, RHP-7-06, RHP-6-06, Colombia XXI, FLAR 179-00, FLAR 195-00, Idiap 54-05 y FLAR 198-00. Los cultivares con mejor estabilidad fueron FLAR 198-00, FLAR 195-00 e Idiap 145-05. Cuando consideramos ambas características (productividad y estabilidad), el cultivar superior fue Idiap 145-05. Fedearroz 473 y Oryzica 1, con altas productividades de grano, presentaron baja estabilidad.

Los resultados sugieren que el cultivar Fedearroz 473 es imbatible en las localidades que componen el mega-ambiente donde muestra su superioridad y responde bien en los ambientes favorables; no obstante, en el otro mega-ambiente, su comportamiento es muy inferior, lo que sugiere que es recomendable para siembras comerciales o como testigo únicamente en el mega-ambiente conformado por las siguientes localidades: Alanje 2006, Calabacito 2007, Tonosí 2007, Barú 2006, Barú 2007, Penonomé 2007, Remedios 2007, Calabacito 2006, Tanara 2006, Soná 2007, Soná 2006, Divisa 2007.

En ambos mega-ambientes, el cultivar Idiap 145-05 puede ser utilizado como una variedad confiable en siembras comerciales; esto sugiere que este cultivar posee adaptación amplia y específica y es un testigo apropiado para todas las localidades que componen este mega-ambiente como: Changuinola 2008, Alanje 08, Soná 2008, Penonomé 08, Tonosí 2008, Barú 08, Alanje 2007, Tanara 07 y Divisa 2008.

La formación de estos mega-ambientes puede deberse a la adaptabilidad amplia de los cultivares superiores Fedearroz 473 e Idiap 145 y no necesariamente debido a la similitud agroclimática entre las localidades agrupadas con cada método. Al comparar los agrupamientos (mega-ambientes) formados durante los tres años de evaluación, se observa que Barú y Soná 2006 y 2007 están ubicados en el mismo grupo; mientras que en 2008 se ubicaron en el otro grupo. Pero, Alanje 2007 y 2008 se ubicaron en el mismo mega-ambiente, y en 2006 estaban ubicados 
en el otro mega-ambiente. Las otras localidades con menos años de evaluación fueron más inestables. Esto se debe posiblemente a las variaciones en el régimen de precipitación pluvial de un año para otro y a la variación en la fecha de siembra, Pacheco et al. (2003) consideran que épocas dentro de un mismo ambiente deben ser tratadas como ambientes distintos. Gauch Junior (1992) destaca que las evaluaciones de similaridad ambiental, basadas en los efectos de la interacción genotipo $\mathrm{x}$ ambiente, son más confiables que aquellas basadas en factores ambientales, a pesar de ser importantes en la producción. La estratificación de los mega-ambientes, según Felipe et al. (2010), puede deberse a la alta adaptabilidad del genotipo vencedor y no necesariamente a la similaridad edafoclimática entre las localidades agrupadas en el mega-ambiente. En relación a la priorización de los ambientes más favorables, para evaluar el germoplasma, sobresalen Soná, Alanje y Barú (Figura 4). De acuerdo con Pacheco et al. (2009), los sitios claves para la selección de genotipos, en las fases iníciales, son las que clasifican a un mayor número de genotipos vencedores.

El agrupamiento de las localidades en mega-ambientes, mediante los tres métodos en este estudio, muestra una coincidencia de $100 \%$ en el agrupamiento del conglomerado $\mathrm{x}$ el biplot GGE, mientras que entre AMMI1 x conglomerado y biplot GGE x AMMI1 fue de 95,2\%. Yan et al. (2007) indican que ambos análisis combinan el análisis de genotipo y la interacción por separado, en la definición de los mega-ambientes y en la selección de cultivares. Ebdon \& Gauch Junior (2002) encontraron que la clasificación de mega-ambientes por el IPC1 puede ser virtualmente similar al basado en biplot GGE. A pesar de que Gauch Junior (2006) afirma que el análisis AMMI separa los efectos de genotipo, ambiente e interacción, antes de la aplicación de la descomposición de valores singulares, esto puede hacerse con cualquier conjunto de datos. El método biplot GGE, (Yan, 2002; Yan et al., 2007) separa los efectos de ambientes antes de la aplicación de la descomposición de valores singulares, después separa el efecto de genotipo y de la interacción genotipo-ambiente, lo que depende de propiedades especiales de la base de datos. Yang et al. (2009) recomiendan a los investigadores considerar que el análisis biplot es simplemente una herramienta estadística descriptiva y visual y que, por tanto, deben proceder con precaución si utilizan el análisis biplot más allá de esta simple función.
Las técnicas de análisis multivariados aplicadas en este estudio mostraron ser efectivas para identificar mega-ambientes, para optimizar el potencial genético de los cultivares de arroz, aunque estos mega-ambientes sean más dependientes de la capacidad adaptativa de los genotipos superiores que de las propias condiciones agroclimáticas de las localidades evaluadas.

\section{Conclusiones}

1. Fedearroz 473 e Idiap 145-05 son los cultivares sobresalientes en cada uno de los dos mega-ambientes identificados.

2. Hay una coincidencia de $100 \%$ en el agrupamiento del conglomerado $\mathrm{x}$ el biplot GGE, mientras que entre AMMI x conglomerado y biplot GGE x AMMI es de $95,2 \%$.

3. El cultivar más estable en ambos mega-ambientes es Idiap 145-05, lo que indica que presenta adaptación específica y amplia.

4. La capacidad adaptativa de los genotipos superiores y no las condiciones agroclimáticas de las localidades evaluadas es responsable por la definición de los mega-ambientes.

\section{Agradecimientos}

Al Instituto de Investigación Agropecuaria de Panamá y al Fondo Regional de Tecnología Agropecuaria, por el apoyo financiero.

\section{Referencias}

ANNICCHIARICO, P.; PECETTI, L.; BOGGINI, G.; DOUST, M.A. Repeatability of large-scale germplasm evaluation results in durum wheat. Crop Science, v.40, p.1810-1814, 2000.

ATLIN, G.N.; BAKER, R.J.; MCRAE, K.B.; LU, X. Selection response in subdivided target regions. Crop Science, v.40, p.7-13, 2000.

BECKER, H.C.; LÉON, J. Stability analysis in plant breeding. Plant Breeding, v.101, p.1-13, 1988.

CROSSA, J.; CORNELIUS, P.L.; YAN, W.K. Biplots of linear-bilinear models for studying crossover genotype $\mathrm{x}$ environment interaction. Crop Science, v.42, p.619-633, 2002.

CROSSA, J.; GAUCH JUNIOR, H.G.; ZOBEL, R.W. Additive main effects and multiplicative interaction analysis of two international maize cultivar trials. Crop Science, v.30, p.493-500, 1990.

EBDON, J.S.; GAUCH JUNIOR, H.G. Additive main effect and multiplicative interaction analysis of national turfgrass performance trials: II. cultivar recommendations. Crop Science, v.42, p.497-506, 2002. 
FELIPE, C.R. de P.; DUARTE, J.B.; CAMARANO, L.F. Estratificação ambiental para avaliação e recomendação de variedades de milho no Estado de Goiás. Pesquisa Agropecuária Tropical, v.40, p.186-199, 2010.

GAUCH JUNIOR, H.G. Statistical analysis of regional yield trials: AMMI analysis of factorial designs. Amsterdam: Elsevier, 1992. 277p.

GAUCH JUNIOR, H.G. Statistical analysis of yield trials by AMMI and GGE. Crop Science, v.46, p.1488-1500, 2006.

GAUCH JUNIOR, H.G.; ZOBEL, R.W. Accuracy and selection success in yield trial analyses. Theoretical and Applied Genetics, v.77, p.473-481, 1989.

GAUCH JUNIOR, H.G.; ZOBEL, R.W. Identifying mega-environments and targeting genotypes. Crop Science, v.37, p. 311-326, 1997.

GLAZ, B.; KANG, M.S. Location contributions determined via GGE biplot analysis of multienvironment sugarcane genotype-performance trials. Crop Science, v.48, p.941-950, 2008.

GORDÓN, R.; CAMARGO, I.; FRANCO, J.; GONZÁLEZ, A. Impacto de la precipitación pluvial en el rendimiento de grano del maíz en la región de Azuero, Panamá, 1995-2003. I. Análisis de la distribución de lluvias y su relación con la época de siembra. Ciencia Agropecuaria, v.16, p.17-30, 2005a.

GORDÓN, R.; CAMARGO, I.; FRANCO, J.; GONZÁLEZ, A. Impacto de la precipitación pluvial en el rendimiento de grano del maíz en la región de Azuero, Panamá, 1995-2003. II. Análisis del rendimiento y su relación con la época de siembra. Ciencia Agropecuaria, v.16, p.31-44, 2005b.

HERNANDEZ, M.V.; CROSSA, J. The AMMI analysis and graphing the biplot. El Batán: CIMMYT, 2000. 42p.

INSTITUTO DE INVESTIGACIÓN AGROPECUARIA DE PANAMÁ. Informe técnico del proyecto de investigación e innovación: desarrollo de germoplasma mejorado de arroz: 2005-2009. Panamá: IDIAP, 2010. 65p.

JOHNSON, D.E. Métodos multivariados aplicados al análisis de datos. Mexico: Thompson, 2000. 566p.

LIN, C.S.; BINNS, M.R.; LEFKOVITH, L.P. Stability analysis. Where do we stand? Crop Science, v.26 p.894-900, 1986.

PACHECO, R.M.; DUARTE, J.B.; ASSUNÇÃO, M. da S.; NUNES JUNIOR, J.; PESSOA CHAVEZ, A.A. Zoneamento e adaptação produtiva de genótipos de soja de ciclo médio de maturação para Goiás. Pesquisa Agropecuaria Tropical, v.33, p.23-27, 2003.

PACHECO, R.M.; DUARTE, J.B.; SOUZA, P.I.M. de; SILVA, S.A. da; NUNES JUNIOR, J. Key locations for soybean genotype assessment in Central Brazil. Pesquisa Agropecuária Brasileira, v.44, p.478-486, 2009.
SAMONTE, S.O.P.B.; WILSON, L.T.; MCCLUNG, A.M.; MEDLEY, J.C. Targeting cultivars onto rice growing environments using AMMI and SREG GGE biplot analyses. Crop Science, v.45, p.2414-2424, 2005.

SAS INSTITUTE. SAS/STAT: users guide. Version 9.0. Cary: SAS Institute, 2002.

TRETHOWAN, R.M.; VAN GINKEL, M.; AMMAR, K.; CROSSA, J.; PAYNE, T.S.; CUKADAR, B.; RAJARAM, S.; HERNANDEZ, E. Associations among twenty years of international bread wheat yield evaluation environments. Crop Science, v.43, p.1698-1711, 2003.

YAN, W. Singular value partitioning in biplot analysis of multienvironment trial data. Agronomy Journal, v.94, p.990-996, 2002.

YAN, W. Visual statistical analysis system GGE biplot pattern explorer. Version 6.3. 2009. Available at: <http://www.ggebiplot. com/FreeGGEbiplot.htm>. Accessed on: 5 Sept. 2011.

YAN, W.; CORNELIUS, P.L.; CROSSA, J.; HUNT, L.A. Two types of GGE biplots for analyzing multi-environment trial data. Crop Science, v.41, p.656-663, 2001.

YAN, W.; HUNT, L.A. biplot analysis of multi-environment trial data. In: KANG, M.S. (Ed.). Quantitative genetics, genomics and plant breeding. Wallingford: CAB International, 2002. p.289-319.

YAN, W.; HUNT, L.A.; SHENG, Q.; SZLAVNICS, Z. Cultivar evaluation and mega-environment investigation based on the GGE Biplot. Crop Science, v.40, p.597-605, 2000.

YAN, W.; KANG, M.S. GGE biplot analysis: a graphical tool for breeders, geneticists, and agronomists. Boca Raton: CRC Press, 2003. 271p.

YAN, W.; KANG, M.S.; MA, B.; WOODS, S.; CORNELIUS, P. GGE biplot vs. AMMI analysis of genotype-by-environment data. Crop Science, v.47, p.643-655, 2007.

YAN, W.; RAJCAN, I. Biplot analysis of test sites and trait relations of soybean in Ontario. Crop Science, v.42, p.11-20, 2002.

YAN, W.; TINKER, N.A. An integrated biplot analysis system for displaying, interpreting, and exploring genotype $\mathrm{x}$ environment interaction. Crop Science, v.45, p.1004-1016, 2005.

YANG, R.-C.; BLADE, S.F.; CROSSA, J.; STANTON, D.; BANDARA, M.S. Identifying isoyield environments for field pea production. Crop Science, v.45, p. 106-113, 2005.

YANG, R.-C.; CROSSA, J.; CORNELIUS, P.L.; BURGUEÑO, J. Biplot analysis of genotype $\mathrm{x}$ environment interaction: proceed with caution. Crop Science, v.49, p.1564-1576, 2009.

ZOBEL, R.W.; WRIGTH, M.J.; GAUCH JUNIOR, H.G. Statistical analysis of a yield trial. Agronomy Journal, v.80, p.388-393, 1988.

Recibido el 13 de abril de 2011 y aprobado el 30 de agosto de 2011

Pesq. agropec. bras., Brasília, v.46, n.9, p.1061-1069, set. 2011 